\title{
THE APRIL MEETING IN LAWRENCE
}

The four hundred forty-seventh meeting of the American Mathematical Society was held at the University of Kansas on Friday and Saturday, April 29-30, 1949. There was a joint session with the Biometric Society on Friday afternoon.

The total attendance was about 150 including the following 84 members of the Society:

L. W. Akers, E. S. Allen, E. W. Anderson, R. W. Babcock, M. A. Basoco, P. O. Bell, Arthur Bernhart, Herman Betz, R. H. Bing, Florence Black, H. D. Block, L. M. Blumenthal, P. B. Burcham, E. D. Cashwell, F. M. Clarke, H. E. Clarkson, H. J. Cohen, M. E. Daniells, Robert Davies, R. F. Deniston, A. H. Diamond, Paul Eberhart, J. H. Engel, G. M. Ewing, W. C. Foreman, R. E. Fullerton, Albert Furman, R. E. Gaskell, R. N. Goss, Cornelius Gouwens, L. Z. Greene, Edison Greer, Edwin Halfar, O. H. Hamilton, P. C. Hammer, W. L. Hart, F. F. Helton, I. N. Herstein, J. J. L. Hinrichsen, D. L. Holl, A. S. Householder, H. K. Hughes, W. C. Janes, L. H. Kanter, M. Z. Krzywoblocki, W. G. Leavitt, H. M. Lieberstein, Anna Marm, M. E. Martinson, E. J. Mickle, J. M. Mitchell, W. K. Moore, D. J. Morrow, Zeev Nehari, J. I. Northam, G. K. Overholtzer, S. T. Parker, M. M. Pihlblad, G. B. Price, O. M. Rasmussen, L. M. Reagan, P. G. Robinson, R. G. Sanger, J. W. Sawyer, Robert Schatten, B. R. Seth, F. B. Sloat, G. W. Smith, H. W. Smith, R. G. Smith, C. E. Springer, W. L. Stamey, R. H. Stark, E. B. Stouffer, W. T. Stratton, H. P. Thielman, R. M. Thrall, J. W. Tukey, Gilbert Ulmer, Bernard Vinograde, M. A. Woodbury, J. L. Yarnell, P. M. Young, J. W. T. Youngs.

By invitation of the Committee to Select Hour Speakers for Western Sectional Meetings, Professor E. J. Mickle of The Ohio State University spoke at 11:00 A.M. Friday on Some properties of the Lebesgue area; and Professor R. M. Thrall of the University of Michigan spoke at 9:00 A.M. Saturday, the title of his address being On classes of algebras with radical.

The joint session with the Biometric Society was at 2:00 P.M. Friday. By arrangement with the program committee the following addresses were given: Dr. A. S. Householder, Oak Ridge National Laboratory, Diffusion of isotopes in biological systems of several compartments; Professor M. A. Woodbury, University of Michigan, Prediction and estimation; Professor J. W. Tukey, Princeton University, The consultant and the biologist.

Sessions for contributed papers were held at 10:00 A.M. Friday and 10:15 A.M. Saturday.

Presiding officers for the various sessions were Professors L. M. Blumenthal, D. L. Holl, E. J. Mickle, G. B. Price, E. B. Stouffer, R. M. Thrall, and Bernard Vinograde.

On Friday evening there was a dinner for the Society in the Me- 
morial Union Building. At the conclusion of the dinner the Society witnessed an excellent production of Hamlet by the Department of Speech and Drama of the University.

Abstracts of all papers presented at the Meeting are given below. Papers read by title are indicated by the letter " $t$. . Paper number 418 was presented by Mr. Fisher and paper number 419 by Dr. Woodbury. Mr. Steel was introduced by Professor Bernard Vinograde.

\section{Algebra ANd Theory of Numbers}

\section{Arthur Bernhart: Roots of polynomials and their derivatives.}

The average product of the roots taken $r$ at a time is the same for the polynomial and all its derivatives. In particular if the roots of a cubic are at the vertices of a triangle, then the quadratic roots are found to be at the foci of the inscribed Steiner ellipse. (Received March 21, 1949.)

\section{F. Marion Clarke: On the factorization of polynomials in $n$ vari- ables. III. \\ For a field $F$ and a polynomial $f\left(x_{1}, \cdots, x_{n}\right)$ in the polynomial domain $F\left[x_{1}, \cdots, x_{n}\right]$ a splitting field $P$ is defined by the properties (i) $P$ is an algebraic ex- tension of $F$, (ii) $f$ is factorable into absolutely irreducible factors over $P$, (iii) there exists no field $P^{\prime}$ properly contained in $P$ and having properties (i) and (ii). It is shown by means of a Kronecker substitution that if $F$ has characteristic zero, $P$ is unique to within relative isomorphism. (Received March 15, 1949.)}

400. J. H. Engel: Contributions to the solution of the word problem for groups.

The word problem for the lower central series of the free group (called hereafter hypo-abelian groups) is solved by a method (distinct from the methods used by Magnus, Fouxe-Rabinovitch, or R. H. Fox) which utilizes invariants connected with a lattice representation of these groups. There is demonstrated for each word in each hypo-abelian group the existence of a unique canonical form, expressed as a product, in canonical order, of powers of generators and powers of commutators. An expansion theorem is developed, proving the known result that elements equalling the identity in all hypo-abelian groups of a free group equal the identity in the given free group. The word problem is solved for hypo-abelian groups of the first order with any number of generators and one additional defining relation, and for hypo-abelian groups of the first or second order with two generators and any number of additional defining relations. These results yield new necessary conditions for the equality of two elements of a group. (Received March 16, 1949.)

\section{I. N. Herstein: The radical of the group ring of a p-group.}

The radical of the group ring of a group $G$ of order $p^{n}$ over a field of characteristic $P$ has been shown to contain all the elements $g-1$ where $g$ is in $G$, by both LombardoRadice and Jennings. In this paper a short proof of this theorem, using elementary concepts of group and ring theory, is given. (Received March 16, 1949.)

402. R. G. D. Steel: $A$ problem on minimum principal minors. 
The problem is to minimize a principal minor of a symmetrically partitioned correlation matrix, one element of the minor from each block, under certain internal congruences, namely, those which preserve the unit diagonal coefficients and which are effected by matrices whose nonzero coefficients are in the blocks corresponding to the partition of the correlation matrix. In order to obtain a system of equations whose solution gives the desired minimum, the procedure is to reduce the problem to orthogonal internal congruences, form a suitable compound and use Cayley's parametrization of the orthogonal group. The result may be considered a generalization of canonical correlations (see H. Hotelling, Relations between two sets of variates, Biometrika vol. 28 (1936) pp. 321-377). (Received March 10,1949.)

\section{3t. A. F. Strehler: The generalization of the Moebius $\mu$-function to a partially-ordered set.}

The familiar Moebius $\mu$-function, originally defined in the set of positive rational integers, is here extended to every partially-ordered set which has the property that for each pair of elements $a, b$ in the set, there is at most a finite number of elements $x$ in the set such that $a \subseteq x \subseteq b$, where $\subseteq$ denotes the relation which orders the set. An explicit formula is found for the function in terms of the internal structure of the set. (Such an extension was made by Weisner, in Trans. Amer. Math. Soc. vol. 38 (1935) pp. 474-484, to a partially-ordered system in which were postulated, in addition to the above condition, the existence of the g. c. d. and l. c. m., relative to $\subseteq$, for each pair of elements of the system.) This extended $\mu$-function is then applied to the inversion of finite series of functions defined over the partially-ordered set; and a generalized Dedekind inversion formula is evolved. In particular, the structure of the $\mu$-function and some applications of the inversion formula are examined in such examples as the set of subclasses of a given finite class, the algebra of statements under the "implication" relation, an arbitrary set of positive rational integers, and integral algebraic domains. (Received March 15, 1949.)

\section{Bernard Vinograde: Algebras generated by every residue system.}

The opposite of a ring which is a direct sum of its radical and semi-simple subring is one which is without this property modulo any proper ideal in the radical. It can be shown that (1) for a ring with minimum conditions the property is equivalent to every complete semi-simple residue system generating the ring, (2) in rings with unit which are primary or commutative or of index less than 4 the property is inherited by subrings $e R e$ where $e$ is any idempotent, (3) for algebras of finite rank the property can be translated into a test for the rank of a matrix easily derived from any multiplication table of the algebra modulo the square of the radical. (Received March 10, 1949.)

\section{Analysis}

405. E. D. Cashwell: The asymptotic solutions of an ordinary differential equation in which the coefficient of the parameter is singular.

The asymptotic solutions of the differential equation $u^{\prime \prime}(z)-\left[\rho^{2} \phi^{2}(z) /\left(z-z_{0}\right)^{2}\right.$ $+\chi(\rho, z)] u(z)=0$ are derived. The variable $z$ ranges over a bounded, simply-connected region of the complex plane which contains the fixed point $z_{0}$, and in which $\phi^{2}(z)$ is bounded from zero. The complex parameter $\rho$ is assumed to be large in absolute value but otherwise unrestricted, while $\chi(\rho, z)$ is analytic in $z$ and uniformly bounded in $\rho$. The procedure depends upon the determination of a differential equation whose solu- 
tions are known, and which approximates the original equation in a specific sense for large $\rho$. Then, for $z$ in a suitably defined closed region containing $z_{0}$ and $\arg \rho$ in each of four nonoverlapping subregions covering the $\rho$-plane, a pair of independent solutions of the initial equation are obtained in terms of the solutions of the approximating equations. The methods used are similar to those of Langer (Trans. Amer. Math. Soc. vol. 37 (1935) pp. 397-416). (Received March 10, 1949.)

406. G. M. Ewing: Surface integrals of the Weierstrass type. Preliminary report.

For each oriented Fréchet surface $S: x(u), u \in R$, of the type of the 2-cell, an area $A(S)$ and a vector $V(S)$ are supposed given, subject to certain postulates. Let $\tau$ be a triangulation of $R$ into triangles $t_{j}$, let $u_{j}$ be a point of $t_{j}$ and let $S_{j}$ be the piece of $S$ represented by $x(u), u \in t_{j}$. If $f(x, r)$ is continuous in $(x, r)$ and homogeneous in $r$ in the usual sense, and if $S$ has suitable properties, the sum $\sum f\left(x\left(u_{j}\right), V\left(S_{j}\right)\right)$ tends to a finite limit, as norm $\tau$ approaches zero, which is independent of the choice of the representation $x(u)$. Lower semi-continuity and convergence properties of the integral are investigated. Connections are found with work of Rado and of Cesari. (Received March 17, 1949.)

407. H. K. Hughes: On the analytical continuation of generalized factorial series. Preliminary report.

The series considered here is $\sum_{n=1}^{\infty} g(n) A_{n}(t, k)$, where $A_{n}(t, k)=k \Gamma(t+k)$ - $\Gamma(n k) /(t+k-1) \Gamma(k) \Gamma(t+n k)$. It reduces to an ordinary factorial series when $k=1$, and to a Dirichlet series when $k \rightarrow \infty$ through appropriate values. (For known results see $T$. Fort, Trans. Amer. Math. Soc. vol. 23, p. 26.) In the present paper it is assumed that the coefficient $g(n)$ becomes an analytic function $g(z)$ when $n$ is replaced by $z=x+i y$, this function being analytic throughout the half-plane $x \geqq 1 / 2$. Two theorems on analytic continuation are obtained. If $g(n)$ has the form $(-1)^{n} h(n)$, where $h(z)$ $=0\{\exp (\pi / 2-\epsilon)|y|\} ; \epsilon>0,|\arg z| \leqq \pi / 2,|z| \rightarrow \infty$, then the function defined by the series can be expressed in a closed form which is analytic for all finite $t$ except those of a certain sequence provided $k$ is not zero or a negative real number. Again, if $g(z)=0\{\exp (\pi-\epsilon)|y|\} ; \epsilon>0,|\arg z| \leqq \pi / 2,|z| \rightarrow \infty$, a similar result holds. (Received March 12, 1949.)

\section{W. G. Leavitt: On vector differential equations.}

Let $A$ be a matrix whose elements and characteristic roots are holomorphic; the vector differential equation $Y^{\prime}=(\lambda A+B) Y$ with $\lambda$ a parameter is then transformable into $W^{\prime}=(\lambda P+Q) W$, where the elements of $P$ below the main diagonal are all zero. For second order equations a further transformation gives diagonal elements of form $\pm \lambda$. Let the upper-right-hand element of $P$ be $\phi ;$ it is shown that a transformation exists replacing $\phi$ by any associate of any member of its residue class modulo $\mu$. Restricting further by the assumption $\mu$ is an associate of $z^{m}(m \geqq 0)$, it then follows that $\phi$ is reducible to one of the $m+1$ canonical forms $0, z^{j}(j=0,1, \cdots, m-1)$. For certain special cases, forms to which the solutions are asymptotic for large $|\lambda|$ are shown to be obtainable. (Received March 9,1949.)

409. Josephine M. Mitchell: An example of a complete double orthogonal system defined on the unit circle. 
We define a system of functions $\left\{\phi_{m n}^{(k)}\right\}\left(m, n=0,1, \cdots ; k=1, \cdots, 2^{n-1}(n \neq 0)\right)$ of class $L^{2}$ in the unit circle $\rho=1$ as follows: $\phi_{m n}^{(k)}(\theta, \rho)=\left(1 / \pi^{1 / 2}\right) \cos m \theta \psi_{n}^{(k)}(\rho)$ ( $m$ even), $=\left(1 / \pi^{1 / 2}\right) \sin m \theta \psi_{n}^{(k)}(\rho)(m$ odd $)$, and $=\left(1 /(2 \pi)^{1 / 2}\right) \psi_{n}^{(k)}(\rho)(m=0)$, where $(\theta, \rho)$ are polar coordinates. The functions $\psi_{n}^{(k)}(\rho)$ are defined analogously to the Haar orthonormal system, namely: $\psi_{0}(\rho)=2^{1 / 2}$ on $0 \leqq \rho \leqq 1, \psi_{n}^{(k)}(\rho)=2^{n / 2}$ on $i_{2 k-1, n}=E\left[\left((2 k-2) / 2^{n}\right)^{1 / 2}<\rho\right.$ $\left.<\left((2 k-1) / 2^{n}\right)^{1 / 2}\right],=-2^{n / 2}$ on $i_{2 k, n}=E\left[\left((2 k-1) / 2^{n}\right)^{1 / 2}<\rho<\left(2 k / 2^{n}\right)^{1 / 2}\right],=0$ on $I-\left(\bar{i}_{2 k-1, n}+\bar{i}_{2 k, n}\right)$, where $I$ is the unit interval $(0,1)$, and at the end points of the intervals $i_{2 k-1, n} i_{2 k, n}$ as equal to the arithmetic mean of the values which it takes in adjacent sub-intervals. It is proved that the system $\left\{\phi_{m n}^{(k)}\right\}$ is orthonormal and complete with respect to functions of class $L^{2}$ in the unit circle. Furthermore the Lebesgue function, $L_{m n}^{(k)}(\alpha, r) \cdot \int_{0}^{1} \int_{0}^{2 \pi}\left|\sum_{\mu, \nu=1}^{m, n} \phi_{\mu \nu}^{(\kappa)}(\alpha, r) \phi_{\mu \nu)}^{(k)}(\theta, \rho)\right| \rho d \rho d \theta\left(\kappa=1, \cdots, 2^{\nu-1}\right)$ is $o(\log m)$ almost everywhere in the unit circle. The following convergence theorem is proved: if $\sum_{m, n=0}^{\infty} a_{m n}^{(k)} \log ^{2}(m+2)<\infty$, then the Fourier-Haar double orthogonal series $\sum_{m, n=0}^{\infty} a_{m n}^{(k)} \phi_{m n}^{(k)}(\theta, \rho)$, converges almost everywhere in the unit circle. (Received March $14,1949$.

\section{C. E. Springer: Union curves and dynamical trajectories.}

In a manifold $V_{n+1}$, a totally geodesic surface $S$ is considered with orientation of $S$ determined by the tangent to a curve $C$ in a hypersurface $V_{n}$ of $V_{n+1}$ and by the first curvature vector in $V_{n+1}$ of the tangent to $C$. The curve $C$ in $V_{n}$ is a union curve with respect to a given congruence of geodesics in $V_{n+1}$ if the surface $S$ along $C$ contains the geodesic of the congruence through each point of $C$. The differential equations of union curves in $V_{n}$ are obtained. A relation connecting union curvature, geodesic curvature, and normal curvature is found. It is shown that the trajectories of a conservative dynamical system of $n$ degrees of freedom correspond to the union curves of a Riemannian space of $n$ dimensions. (Received March 16, 1949.)

\section{H. P. Thielman: On functional equations and generalized means.}

The mean $M_{f}[F]$, with respect to a strictly monotone function $f(x)$ and a finite distribution function $F(x)$ is defined as in the book Inequalities by Hardy, Littlewood and Polya. Just as thc powers of $x$ lend themselves readily to the definition of a continuous set of means, it is shown that the Tschebyscheff polynomials can be used in a similar way. Means with respect to $\cos f \operatorname{arc} \cos x$ are defined for finite sets of numbers over subintervals of $0 \leqq x \leqq 1$. Some theorems given in the above mentioned book for means with respect to $x^{p}$ have their analogues in the theory given here. Necessary and sufficient conditions on a polynomial $F(x, y)$ are given in order that the functional equation $\phi[F(x, y)]=\phi(x)+\phi(y)$ can have monotone continuous solutions. (Received March 15, 1949.)

\section{Applied Mathematics}

\section{R. N. Goss: Center of flexures of a triangular beam.}

In Saint-Venant's flexure problem the center of flexure is defined as that point of loading for which the local twist vanishes at the centroid of a section. Using Seth's solution of the flexure problem for a beam of isosceles-triangular section (Proc. London Math. Soc. (2) vol. 41 (1936) pp. 323-331), the center of flexure is determined for any isosceles triangle for any value of Poisson's ratio. Previously known results for the right isosceles and incompressible equilateral triangles are included as special cases. Excellent agreement is obtained with the experimental results of Duncan, Ellis and Scruton on a very narrow section (Philosophical Magazine (7) vol. 16 (1933) 
pp. 201-235). In the equilateral triangle the position of the center of flexure is found to depend on Poisson's ratio if resultant rather than exact boundary conditions are imposed on two of the sides. (Received April 25, 1949.)

\section{M. Z. Krzywoblocki: The invariant theory of isotropic, homo- geneous turbulence in compressible fluid.}

Robertson's invariant theory of isotropic turbulence valid for an incompressible fluid was adjusted to a compressible flow. The correlation tensors are not any more solinoidal tensors. All the correlation tensors of the second and third rank are derived as well as the dynamical equations of motion and energy. (Received March 5,1949.)

\section{B. R. Seth: Boundary conditions interpreted as conformal transformation.}

Solutions of two-dimensional problems in which a liquid is partly bounded by fixed plane walls and partly by surfaces of constant pressure are obtained by interpreting the boundary condition as a conformal transformation of a rectilinear area in the $z$-plane into a rectilinear area in another z-plane. Similarly all problems of irrotational flow either inside or outside a rectilinear polygonal cylinder can be solved by transforming the boundary condition into a form such that it amounts to only a conformal transformation from one plane to another (Seth, Philosophical Magazine vol. 20 (1935) pp. 632-640; Quarterly Journal of Mathematics vol. 5 (1934) pp. 161-171; Hodgkinson, Quarterly Journal of Mathematics vol. 6 (1935) pp. 171-183). This method has also been applied to a number of torsion and flexure problems in elasticity and can be easily extended to problems in viscous flow, electricity and magnetism and conduction of heat (Trefftz, Math. Ann. vol. 82 (1921) pp. 97-112; Seth, Proc. Cambridge Philos. Soc. vol. 33 (1934) pp. 392-403; Philosohical Magazine vol. 22 (1936) pp. 582-598; vol. 23 (1937) pp. 745-757). It has also been used for cylinders bounded by confocal parabolas (Seth, Proceedings of the Indian Academy of Sciences vol. 9 (1939) pp. 447-453). It is proposed to extend it to curvilinear boundaries. (Received March 17, 1949.)

\section{5t. D. M. Studley: The mathematics of language.}

As point of departure from conventional philosophizing the notion of Plato's Forms as sets is taken and raised to the level of a universal principle. Seen in that light ideas or words become fair game for set-theoretic manipulation. In a case such as that of the Form of Whiteness it seems fully natural to think of the class of white things and objects as typically representing the abstract form White. Extending the inspection to other colors, a set of primary colors can be selected. The union of red and blue is purple while the union of yellow and red is orange. Orange and purple intersect in red. Such application is truly endless and quite inescapable because thought with words has unique pattern. Since a word generally has a set of senses, some of which may intersect, a word-form is a set of classes. In event of ambiguity of meaning doubt is founded upon union of possible meanings. Clearly set addition is mostly non-commutative since meaning of words depends on their order although combinations sometimes occur where over-all meaning is not changed by change of order. A sentence is a complete set of words whereas a description is an incomplete set of words. Nevertheless a one sentence description is possible and occurs in the use of language with an indefinite frequency. Notion $\in$ words and meaning $C$ word $C$ sentence $C$ abstract. 
The result of this method has been the construction of a logical semantics. (Received March 16, 1949.)

\section{GEOMETRY}

\section{P. O. Bell: On hypergeodesic triangles the ratio of those integral curvature to angular excess is constant.}

A new system of hypergeodesics $G_{k}$ (generalized geodesics) is characterized on a general analytic surface $S$ by the property that on any triangle $T$ formed by arcs of the system $G_{k}$ the ratio of the integral curvature of $T$ to the "angular excess" of $T$ is equal to $k$, a constant. The geodesics of $S$ constitute the special system $G_{1}$. Let $e$ denote a unit tangent vector at a point $x$ of a curve $C_{\lambda}$ of the system $G_{k}$ and let $d s$ denote the element of arc length of $C_{\lambda}$ at $x$. Let $C_{1}, C_{2}$ denote the curves passing through $x$ of an arbitrary orthogonal net $N$ of $S$, and let $e_{1}, e_{2}$ and $d s_{1}, d s_{2}$ denote the unit tangent vectors at $x$ and the elements of arc length $C_{1}, C_{2}$, respectively. The components of vector curvature $d e / d s$ normal to the surface and tangent to the surface are $k_{n} n$ and $\gamma e^{*}$, respectively, where $k_{n}$ is the normal curvature of $S$ for the direction $\lambda, \gamma$ is the geodesic curvature of $C_{\lambda}$ at $x$, and $e^{*}$ is the unit tangent vector perpendicular to $e$ such that $e^{*} \times e=n$. The geometric characterization of the vector curvature $d e / d s$ is completed on establishing the following result: The geodesic curvature $\gamma$ of a hypergeodesic of the system $G_{k}$ is given by the scalar product $\gamma=(k-1)$ $\cdot\left(d e_{1} / d s_{1}+d e_{2} / d s_{2}\right) \cdot e^{*}$ in which $d e_{1} / d s_{1}$ and $d e_{2} / d s_{2}$ are the vector curvatures of $C_{1}$ and $C_{2}$, respectively. (Received March 16, 1949.)

\section{7t. John DeCicco: General geometry of second order elements.}

The group of arbitrary point transformations of the plane induces a certain eight parameter group $G_{8}$ on the differential elements of second order, all of which pass through a given point $(x, y)$. In 1910, Kasner obtained all the invariants under this $G_{8}$ for $n$ such elements. In the present paper, the effect of this $G_{8}$ upon the central locus $C$ corresponding to a fixed point $(x, y)$ of a second order differential equation is studied. It is proved that under $G_{8}$ an arc length $s$ exists for $C$ involving derivatives of fifth order. The curvature $\kappa$ of $C$, invariant under $G_{8}$, is an expression involving derivatives of seventh order. Any two such curves whose curvatures $K$ are the same functions of the arc length $s$ are equivalent under $G_{8}$. (Received December 27, 1948.)

418. R. C. Fisher and P. O. Bell: New geometric characterizations for the lines of curvature and the geodesics of a surface.

Let $\pi_{1}$ and $\pi_{2}$ denote the normal planes to an analytic surface $S$ in ordinary space at a generic point $x$ in the directions of the $u$ and $v$ parametric curves respectively. The planes $\pi_{1}$ and $\pi_{2}$ generate developable surfaces as $x$ moves along an arbitrary curve $C_{\lambda}$ of $S$. Let $P_{\lambda}$ and $Q_{\lambda}$ denote the intersections of the normal to $S$ at $x$ with the characteristic lines of $\pi_{1}$ and $\pi_{2}$ for the direction $\lambda$. The points $P_{\lambda}$ and $Q_{\lambda}$ coincide for an arbitrary parametric net if and only if the curve $C_{\lambda}$ is a line of curvature. Let $\pi_{\lambda}$ denote the normal plane in the direction $\lambda$. The intersections of the characteristic lines of $\pi_{\lambda}$ for the $u$ and $v$ directions with the normal (tangent plane) coincide if and only if the curve $C_{\lambda}$ is a line of curvature (geodesic). If $C_{\lambda}$ and $C_{\lambda}^{-}$are curves of a net on $S$, any two of the following conditions implies the third: (a) $C_{\lambda}$ and $C_{\lambda}^{-}$intersect at a constant angle of $S$; (b) $C_{\lambda}$ is a geodesic; (c) the characteristic line of $\pi \bar{\lambda}$ for the direction $\lambda$ is parallel to the tangent plane at $x$. (Received March 16, 1949.) 


\section{Statistics and Probability}

419. C. L. Dolph and M. A. Woodbury: An application of the Carleman singular integral equation theory to the spectral representation of stochastic processes. Preliminary report.

Implicit in Carleman's work is a generalization of Mercer's theorem to positive definite Hermitian kernels of his class I (that is, where the associated integral operator $\int \rho(s, t)() d t$ is hypermaximal) which reduces to a form of Bochner's theorem when $\rho(x, t)=\rho(s-t)$. This observation leads to a spectral representation of processes $\xi(x \mid t)$ whose correlation function $\rho(s, t)=\mathrm{E}[\xi(x \mid s) \overline{\xi(x \mid t)}]$ is of Carleman's class I. This parallels and generalizes the Cramer-Karhunen representation of stationary processes. This representation does not seem to satisfy Karhunen's definition of a spectral representation but is similar in many respects. (Received February 19,1949.)

420. J. I. Northam: Single and double dependent rare events. Preliminary report.

Let $n=n_{1}+2 n_{2}$ where $n_{1}$ and $n_{2}$ are respectively the number of occurrences of certain single and double rare events, and where $n_{1}$ and $n_{2}$ are assumed to follow a bivariate Poisson distribution with parameters $\lambda_{10}, \lambda_{01}, \lambda_{11}$. The probability generating function for $P(n)$ is found by use of the probability generating function for a bivariate Poisson distribution. Previously, Cernuschi and Castagnetto (Ann. Math. Statist. vol. 17, no. 1) found $P(n)$ for $n=n_{1}+2 n_{2}+\cdots+i n_{i}+\cdots$ where the $n_{i}$ were assumed to follow independent Poisson laws with $\lambda_{i}=\lambda_{1} a^{8-1} / s !, a \geqq 0$. (Received March 7, 1949.)

\section{TOPOLOGY}

\section{R. H. Bing: Metrization of topological spaces.}

A collection of point sets is discrete if the closures of these point sets are mutually exclusive and any subcollection of these closures has a closed sum. Generalizing the notion of perfect separability, we say that space is perfectly screenable if there exists a sequence $G_{1}, G_{2}, \cdots$ such that $G_{i}$ is a discrete collection of domains and for each domain $D$ and each point $p$ in $D$ there is an integer $n(p, D)$ such that $G_{n(p, D)}$ contains a domain which lies in $D$ and contains $p$. A regular topological space is metrizable if and only if it is perfectly screenable. Space is screenable if for each open covering $H$ of space, there is a sequence $H_{1}, H_{2}, \cdots$ such that $H_{i}$ is a collection of mutually exclusive domains and $\sum H_{i}$ is a covering of space which is a refinement of $H$. While a screenable Moore space need not be normal, it is metrizable if it is normal. Space is collectionwise normal if for each discrete collection $X$ of closed point sets, there is a collection $Y$ of mutually exclusive domains covering $X^{*}$ such that no element of $Y$ intersects two elements of $X$. A Moore space is metrizable if it is collectionwise normal. (Received March 14, 1949.)

\section{H. J. Cohen: Homogeneous plane continua.}

In 1924, S. Manzurkiewicz proved that a homogeneous, bounded, plane continuum $M$ which is locally connected must be a simple closed curve. The existence of a homogenous, bounded, plane continuum other than a simple closed curve has been established by R. H. Bing (1948). The present paper strengthens the result of Mazurkiewicz by showing, first, that it holds even if $M$ is locally homogeneous-that is, for each 
pair of its points $x, y$, there exists a homeomorphism between two open subsets of $M$, one containing $x$, the other containing $y$, such that $x$ maps into $y$. Alternatively, the requirement of local connectedness can be replaced by the condition that $M$ contain a simple closed curve. It is incidentally shown that any bounded, plane continuum which is the sum of a collection of disjoint simple closed curves is topologically equivalent to an annulus. (Received March 9, 1949.)

423. O. H. Hamilton: Transformations topologically equivalent to isometric transformations in Hilbert space.

It is shown that if $T$ is a pointwise periodic transformation of a point set in an $n$-dimensional Euclidean space, then $T$ is topologically equivalent to a transformation of a point set in a Hilbert space which leaves the distance between two points invariant. (Received March 17, 1949.)

J. W. T. Youngs, Associate Secretary

\section{THE APRIL MEETING IN STANFORD UNIVERSITY}

The four hundred forty-eighth meeting of the American Mathematical Society was held at Stanford University, Palo Alto, California, on Saturday, April 30, 1949. The attendance was approximately 85, including the following 71 members of the Society:

M. I. Aissen, T. M. Apostol, H. A. Arnold, H. M. Bacon, E. M. Beesley, R. L. Belzer, H. F. Bohnenblust, J. L. Botsford, F. A. Butter, Lamberto Cesari, C. L. Clark, F. G. Creese, P. H. Daus, A. C. Davis, E. A. Davis, M. R. Demers, S. P. Diliberto, Roy Dubisch, W. D. Duthie, J. M. G. Fell, G. E. Forsythe, A. L. Foster, R. A. Fuchs, P. R. Garabedian, M. A. Girshick, J. W. Green, Sarah Hallam, G. J. Haltiner, M. A. Heaslet, J. G. Herriot, M. R. Hestenes, R. E. Holdman, R. D. James, Samuel Karlin, J. L. Kelley, R. M. Lackness, Cornelius Lanczos, D. H. Lehmer, Hans Lewy, Eugene Lucacs, Rhoda Manning, W. A. Mersman, A. B. Mewborn, B. C. Meyer, E. D. Miller, F. R. Morris, Ivan Niven, A. M. Ostrowski, Anna Pell-Wheeler, R. S. Phillips, Edmund Pinney, Hugo Ribiero, J. B. Robinson, R. M. Robinson, H. L. Royden, Wladimir Seidel, Max Shiffman, D. C. Spencer, W. M. Stone, Irving Sussman, J. D. Swift, L. H. Swinford, Gabor Szegö, J. L. Ullman, F. A. Valentine, R. L. Vaught, Morgan Ward, D. V. Widder, V. A. Widder, A. R. Williams, František Wolf.

In the morning there was a general session for research papers and for the invited address, Quadratic forms in the calculus of variations, by Professor M. R. Hestenes of the University of California, Los Angeles. Professor Max Shiffman presided. In the afternoon there were two sections, pure and applied mathematics, at which Professors D. C. Spencer and Gabor Szegö presided.

After the meetings, those attending were guests at a tea in Stanford Union. 